

REVISÃO BIBLIOGRÁFICA

Recebido em: 11/2020

Aceito em: $12 / 2020$

Publicado em: 1/2021

\title{
Infecções sexualmente transmissíveis entre acadêmicos da área da saúde
}

\author{
Sexually transmitted infections among health academics
}

Infecciones de transmisión sexual entre académicos de la salud

Rejane Pereira ${ }^{1}$, Maria Adriely Cunha Lima ${ }^{1 *}$, Joéliton Gois Silva ${ }^{1}$, Tiago Almeida Costa ${ }^{1}$, Tatiane de Oliveira Santos ${ }^{1}$, Veida Borges Soares de Queiroz ${ }^{1}$, Mariana Souza Tavares Santos ${ }^{1}$, Sabrina Barreto Antunes ${ }^{1}$, Tatiane dos Santos ${ }^{2}$, Halley Ferraro Oliveira ${ }^{1}$.

\begin{abstract}
Resumo: $O$ artigo procurava analisar o conhecimento e a atitude entre acadêmicos da área da saúde sobre as Infecções Sexualmente Transmissíveis (IST's). Trata-se de uma revisão integrativa de literatura, para a qual foram utilizados artigos publicados entre 2010 e 2020, pesquisados a partir das bases de dados BVS, MEDLINE, LILACS, SciELO e BDENF. Nas áreas de saúde evidenciou que os acadêmicos possuem conhecimentos sobre as IST's, conhecimentos esses aos quais incluem as formas de prevenção e as consequências; sendo que, mesmo possuidores de conhecimentos ainda assim praticam relações sexuais de forma desprotegida, ficando vulneráveis a se infectar. Nota-se que é imprescindível a informação e o conhecimento para a devida prevenção das contaminações, porém não basta estar ciente da necessidade de se proteger perante as IST's, é preciso que os acadêmicos de saúde não hesitem em se prevenir nas relações sexuais. Dessa forma, não só é importante a conscientização, mas ações efetivas de proteção, uma vez que os acadêmicos são detentores de conhecimento na área.
\end{abstract}

Palavras-chave: Doenças sexualmente transmissíveis, Prevenção de doenças transmissíveis, Estudantes.

\begin{abstract}
The article sought to analyze the knowledge and attitude among health academics about Sexually Transmitted Infections (STIs). It is an integrative literature review, for which articles published between 2010 and 2020 were used, searched from the VHL, MEDLINE, LILACS, SciELO and BDENF databases. In the health areas, it was evidenced that academics have knowledge about STIs, knowledge which includes the forms of prevention and the consequences; even though they have knowledge, they still practice unprotected sex, becoming vulnerable to becoming infected. It is noted that information and knowledge is essential for the proper prevention of contamination, but it is not enough to be aware of the need to protect yourself against STIs, it is necessary that health academics do not hesitate to prevent themselves in sexual relations. Thus, awareness is not only important, but effective protection actions, since academics have knowledge in the area.
\end{abstract}

Keywords: Sexually transmitted diseases, Communicable disease prevention, Students.

\footnotetext{
${ }^{1}$ Universidade Tiradentes (UNIT), Aracaju - SE. *E-mail: mariaadrielycunha@hotmail.com

${ }^{2}$ Centro Universitário Estácio de Sá (ESTÁCIO), Aracaju - SE.
} 
Resumen: El artículo buscó analizar el conocimiento y la actitud de los académicos de la salud sobre las Infecciones de Transmisión Sexual (ITS). Se trata de una revisión de la literatura integradora, para la cual se utilizaron artículos publicados entre 2010 y 2020, buscados en las bases de datos VHL, MEDLINE, LILACS, SciELO y BDENF. En las áreas de salud, se evidenció que los académicos tienen conocimientos sobre las ITS, conocimientos que incluyen las formas de prevención y las consecuencias; a pesar de que tienen conocimiento, todavía practican sexo sin protección, volviéndose vulnerables a infectarse. Se advierte que la información y el conocimiento es fundamental para la adecuada prevención de la contaminación, pero no basta con ser consciente de la necesidad de protegerse contra las ITS, es necesario que los académicos de la salud no duden en prevenirse en las relaciones sexuales. Así, la conciencia no solo es importante, sino acciones de protección efectivas, ya que los académicos tienen conocimientos en el área.

Palabras clave: Enfermedades de transmisión sexual, Prevención de enfermedades transmisibles, Estudiantes.

\section{INTRODUÇÃO}

Infecções Sexualmente Transmissíveis (IST's) são transmitidas pelo contato sexual por via oral, vaginal e/ou anal sem o uso de preservativo com uma pessoa infectada. Essas infecções são causadas por vírus, bactérias e microrganismos que se instalam no paciente infectado. Ademais, as IST's podem ser transmitidas de outras maneiras como: transmissão vertical, amamentação ou por contato com secreções corporais contaminadas, entretanto o risco de contaminação por essas formas é reduzido (BRASIL, 2015).

As IST's contêm diversas formas de apresentação clínica e são responsáveis por interferirem de forma negativa na qualidade de vida das pessoas e nas relações sociais, logo nota-se a importância das políticas públicas destinadas para prevenção dessas doenças. Além disso, as IST's são responsáveis por inúmeras consequências como, esterelidade, malformação fetal, impotência, óbito fetal e outras, caso não sejam tratadas de forma correta, por isso a importância da assistência de saúde para o diagnóstico precoce e o tratamento. Por se tratar de doenças transmissíveis, é importante tratar corretamente os indivíduos diagnosticadas com IST e todos os seus parceiros sexuais, de modo haja interrupção da cadeia de transmissão, além da prevenção das complicações e de outras infecções (BRASIL, 2015).

Infecções Sexualmente Transmissíveis são consideradas como um dos problemas de saúde pública mais comuns em todo o mundo. Sabe-se que o indivíduo que possue alguma infecção classificada como IST's, está mais vulnerável a adquirir outras infecções do mesmo grupo. Ademais, há também uma relação desse grupo de doencas com o aumento da mortalidade materna e infantil (SÁFADI MAP, 2018; SILVA AR e PADILHA MI, 2016). Elas abrangem um diverso grupo de afecções das quais destacam-se, por exemplo, infecção pelo vírus da imunodeficiência humana (HIV), algumas formas de hepatite e sífilis. Estas, relacionamse mais com complicações graves e pior prognóstico.

No Brasil, há grande prevalência da associação entre IST sintomática e assintomática, sendo que há destaque para as infecções de etiologia virais, que são responsáveis por $41,9 \%$ dos casos, enquanto apenas 14,4\% são causadas por bactérias (BRASIL, 2015).

No caso dos adolescentes e jovens, merece destaque o papilomavírus humano (HPV) que tem uma alta prevalência, sugerindo que a infecção ocorra no início das relações sexuais, além das infecções por clamídia e gonococo (BRASIL, 2019).

No que tange a infecção pelo HIV, houve registro de 42.420 novos casos em 2017 no Brasil com predomínio na faixa etária de 20 a 29 anos (PEREIRA GFM, et al., 2019). Hodiernamente, as IST's são frequentemente apontadas como um fator de risco adicional, pelo fato delas deixarem o organismo mais vulnerável a fatores exógenos e, consequentemente, acabam sendo um facilitador no processo de transmissão do HIV (SILVA AR e PADILHA MI, 2016).

É válido salientar a questão da exclusão social de homems homossexuais, mulheres transsexuais e mulheres profissionais do sexo impacta até no acesso aos serviços de saúde, dado isso há uma dificuldade 
na prevenção e no tratamento desses indivíduos que compõem a população de maior risco para infecção pelo HIV (PEREIRA, 2019).

Referente ao período do ciclo de vida, sabe-se que as IST's prevalecem entre os adolescentes e os jovens, especialmente, pelo fato de serem socialmente e sexualmente mais ativos, pois estão em fase de descobrimento do corpo, contato com novas pessoas, por exemplo. Isso aponta a importância de se trabalhar a sensibilização e a prevenção nessa faixa etária (TEIXEIRA RC, et al., 2018).

É válido salientar que a puberdade é um evento que ocorre durante a adolescência causando muitas modificações no corpo. Nesse mesmo tempo, os adolescentes iniciam a vida sexual, sendo que isso tem acontecido mais precocemente. Esse início, na maioria das vezes, não planejado, sem orientação e sem uso de método contraceptivo tem resultado em situações de saúde que podem afetar as suas vidas, principalmente a saúde sexual e reprodutiva, contribuindo para aquisição de IST's (SILVA SPC, et al., 2018).

Torna-se necessário ressaltar a importância do conhecimento sobre as IST's no meio dos jovens e adolescentes, para assim reduzir as consequências e tornar as formas de prevenção dessas infecções atitudes frequentes (ALMEIDA LCG, et al., 2020).

Em relação aos estudantes dos cursos de saúde, Sales WB, et al. (2016), em seu estudo com 819 universitários dos cursos de Biomedicina, Enfermagem, Farmácia, Fisioterapia, Nutrição, Educação física e Psicolgia relatou que $62,8 \%$ dessa amostra não utilizavam o preservativo masculino ou feminino em suas relações sexuais. Dentre os cursos, destacou-se maior risco para os estudantes da Biomedicina e, a cerca do conhecimento desses estudantes sobre as IST's obteve-se uma taxa de 98\% para a Síndrome da imunodeficiência adquirida (SIDA), enquanto que outras afecções como a Infecção pelo Vírus T-linfotrópico humano (HTLV) e a donovanose eram conhecidas por menos de $25 \%$ dos estudantes. Ademais, sabe-se que a realidade de infecção não é diferente entre os acadêmicos da área da saúde, principalmente entre os mais jovens, pois mesmo com o conhecimento que os discentes agregam na universidade, faltam ações efetivas de prevenção por parte dos mesmos.

No que diz respeito aos acadêmicos de enfermagem, um estudo descritivo realizado por Silva BB, et al. (2018) observou-se que eles apresentam um bom conhecimento prévio acerca das IST's, por exemplo, 93\% dos participantes sabiam todas as vias de transmissão e sobre a necessidade de tratamento do parceiro sexual. O estudo também destaca a importância desses indivíduos terem conhecimento sobre essas doenças, visto que futuramente iram disseminar informações em serviços de saúde.

É válido mencionar a importância do conhecimento dos estudantes da área da saúde acerca das IST's devido sua atuação como multiplicadores, ou seja, indivíduos que participam de um grupo e se aprofundam em temas de modo que repassem o conhecimento adquirido para outras pessoas. O estudo de Murad ACS, et al. (2018) aborda isso, visto que por meio de acadêmicos de medicina que atuaram como multiplicadores, eles realizaram uma dinâmica para propor conhecimento a jovens sobre IST's abordando temas como HIV, hepatites virais e a transmissão vertical da sífilis. Além disso, os acadêmicos tentaram juntos com esses jovens identificar situações de risco e de vulnerabilidade em que eles se expõem, a fim de realizar aconselhamento para cessá-las. Silva BB, et al. (2018) também utilizou multiplicadores para avaliar se haveria mudança entre o conhecimento prévio e o após a intervenção deles nos acadêmicos de enfermagem, a partir dos resultados ficou evidente a efetividade dessa estratégia, visto que houve agregação de novas informações, por exemplo, após a atividade realizada com os multiplicadores, $100 \%$ dos participantes sabiam todas as vias de transmissão e sobre a necessidade de tratamento do parceiro sexual.

A justificativa de interesse pela temática da presente pesquisa foi motivada pelo elevado número de IST's em adolescentes e adultos jovens no Brasil, as quais compõem grande parte dos estudantes da saúde. Além disso, sabe-se que esse tema é bastante discutido durante a graduação, surgindo uma inquietação em investigar se há discrepância entre o conhecimento e as atitudes dos acadêmicos da área de saúde. Assim, este trabalho tem como objetivo analisar o conhecimento e a atitude entre acadêmicos da área da saúde sobre as IST's, haja vista que para esse público, em geral, há atividade sexual frequente e um conhecimento prévio acima do senso comum sobre a temática, porém que pode não se refletir na prática da prevenção. 


\section{MÉTODOS}

Trata-se de uma revisão integrativa de literatura, com abordagem descritiva, a qual compreende e proporciona por meio do método de síntese de conhecimento, a aplicabilidade de resultados de estudos significativos. Compreende-se seis fases para o processo de elaboração da revisão integrativa: elaboração de pergunta norteadora, busca ou amostragem na literatura, coleta de dados, análise crítica dos estudos incluídos, discussão dos resultados e apresentação da revisão integrativa (SOUZA TS, et al., 2010).

O estudo foi iniciado a partir da seguinte questão norteadora: qual o conhecimento e atitudes dos acadêmicos da área de saúde em relação as IST's, estão evidenciados na literatura especializada? O período de realização foi de janeiro a junho de 2020. A busca se deu nas seguintes bases de dados nacionais e internacionais, Biblioteca Virtual em Saúde (BVS), Medical Literature Analysisand Retrieval Sistem online (MEDLINE), Literatura Latino-americana e do Caribe em Ciências da Saúde (LILACS), Scientific Eletronic Library Online (SciELO) e Base de Dados de Enfermagem (BDENF).

A busca nas bases de dados deu-se a partir dos seguintes descritores em ciência da saúde (DeCS) "Infecção Sexualmente Transmissíveis" (D1), "Conhecimento" (D2), "Prevenção" (D3), "Preservativos" (D4), "estudantes" (D5), que foram utilizados com o operador booleano "AND" da seguinte forma: "D1" AND "D2" AND "D3" e "D1" AND "D4" AND "D5".

Foi utilizado como critérios de inclusão, artigos originais que tratem do tema publicados na íntegra nos últimos 10 anos, inclusive os que discutiam sobre a visão dos jovens e dos adolescentes de forma que fosse possível atingir o objetivo proposto para o presente trabalho. Já como critérios de exclusão foram artigos em duplicidade, trabalhos de conclusão de curso, cartas aos editores e cartilhas do Ministério da Saúde.

Como resultados da busca foram encontrados 2.776 artigos, com a aplicação dos filtros para que fossem alcançados os critérios de inclusão e exclusão o quantitativo passou a ser 104, dessa forma, a seleção de publicações foi realizada de acordo com os títulos e resumos que atendessem aos objetivos e propósitos da pesquisa, com isso apenas 10 artigos foram selecionados como repostas a questão norteadora. Após leitura de todos os artigos selecionados, o roteiro de coleta de dados foi disposto na Tabela 1.

Tabela 1 - Dados sobre a pesquisa nas determinadas bases de dados.

\begin{tabular}{ccc}
\hline Base de dados & Artigos encontrados & Artigos inclusos \\
\hline LILACS & 60 & 3 \\
\hline MEDLINE & 18 & 2 \\
\hline BDENF & 26 & 2 \\
\hline SCIELO & 4 & 1 \\
\hline BVS & 14 & 2
\end{tabular}

Fonte: Pereira R, et al., 2020.

\section{RESULTADOS E DISCUSSÃO}

A partir da busca na literatura por artigos que respondessem à questão norteadora da presente pesquisa foram encontrados um total de 10 artigos conforme descrito no Quadro 1. Dessa forma, foram destacados 8 artigos sobre a temática abordada com detalhamento de seus objetivos e resultados.

A adolescência é, segunto vários autores, um período de transição (infância e fase adulta) marcado por crescimento, amadurecimento e desenvolvimento, em que há grande aprendizagem, já que há presente vários contextos durante o convívio social na escola e na família, além disso há o o exercício da sexualidade e afetividade, em que eles relacionam-se e interagem (CORTEZ EA e SILVA LM, 2017). No que diz respeito a sexualidade, os adolescentes são influenciados pelas crenças e pelos valores pessoais e familiares, além dos seus pretextos e normas morais. 
Quadro 1- Artigos selecionados em relação à questão norteadora da pesquisa.

\begin{tabular}{|c|c|c|c|c|}
\hline Título & Autores & Periódicos & Objetivos & Resultados \\
\hline $\begin{array}{l}\text { Pesquisa-ação: Promovendo } \\
\text { educação em saúde com } \\
\text { adolescentes sobre Infecção } \\
\text { Sexualmente Transmissivel }\end{array}$ & $\begin{array}{l}\text { CORTEZ EA e } \\
\text { SILVA LM }\end{array}$ & $\begin{array}{l}\text { Reven Ferm } \\
\text { UFPE }\end{array}$ & $\begin{array}{l}\text { Buscar sobre ações de } \\
\text { educação como forma de } \\
\text { prevenção das IST com } \\
\text { adolescentes }\end{array}$ & $\begin{array}{c}\text { Entre as ações importantes para se realizar no âmbito da escola } \\
\text { estão: palestras sobre IST's realizada por profissionais de saúde } \\
\text { na escola, diálogo permanente entre servidores para dinamizar } \\
\text { as IST's e rodas de conversas com os adolescentes sobre } \\
\text { sexualidade }\end{array}$ \\
\hline $\begin{array}{l}\text { Experiência de acadêmicos } \\
\text { de Medicina na Prevenção de } \\
\text { Infecções Sexualmente } \\
\text { Transmissíveis }\end{array}$ & $\begin{array}{c}\text { MURAD ACS, et } \\
\text { al. }\end{array}$ & $\begin{array}{l}\text { Cuid. Arte } \\
\text { Enfermagem }\end{array}$ & $\begin{array}{l}\text { Procurar informações sobre o } \\
\text { uso da experiência de } \\
\text { acadêmicos de medicina como } \\
\text { forma de prevenção das IST }\end{array}$ & $\begin{array}{c}\text { O compartilhamento de informações sobre as IST's com os } \\
\text { grupos mais vulneráveis é uma iniciativa que colabora de forma } \\
\text { positiva para a prevenção com aqueles que não detém } \\
\text { determinado conhecimento }\end{array}$ \\
\hline $\begin{array}{l}\text { Vulnerabilidade de } \\
\text { Adolescentes às Doenças } \\
\text { Sexualmente Transmissíveis } \\
\text { na Atenção Primária }\end{array}$ & $\begin{array}{c}\text { OLIVEIRA PS, et } \\
\text { al. }\end{array}$ & $\begin{array}{l}\text { Rev. Enferm } \\
\text { UFPE }\end{array}$ & $\begin{array}{l}\text { Analisar os aspectos que } \\
\text { favorecem o público jovem a } \\
\text { estar expostos as IST }\end{array}$ & $\begin{array}{l}\text { Os adolescentes em relação à sexualidade, sofre influências } \\
\text { de crenças e valores pessoais e familiares, além disso é } \\
\text { crescente o número de adolescentes que tem a primeira relação } \\
\text { precocemente, o que acarreta em uma maior vulnerabilidade às } \\
\text { IST's }\end{array}$ \\
\hline $\begin{array}{l}\text { Infecções Sexualmente } \\
\text { Transmissíveis na } \\
\text { Adolescência. }\end{array}$ & SÁFADI MAP & $\begin{array}{l}\text { Sociedade } \\
\text { Brasileira de } \\
\text { Pediatria }\end{array}$ & $\begin{array}{c}\text { Identificar informações acerca } \\
\text { de fatores de risco e } \\
\text { prevenções das IST na } \\
\text { adolescência }\end{array}$ & $\begin{array}{l}\text { Comportamentos de risco e fator de vulnerabilidade dos } \\
\text { adolescentes, o uso do álcool e de outras drogas, } \\
\text { frequentemente utilizadas, causando prejuízos de forma direta à } \\
\text { saúde aumentando assim a vulnerabilidade as IST's }\end{array}$ \\
\hline $\begin{array}{l}\text { Acadêmicos de Enfermagem } \\
\text { e seu autocuidado em relação } \\
\text { a Doenças Sexualmente } \\
\text { Transmissíveis }\end{array}$ & $\begin{array}{l}\text { SILVA AR e } \\
\text { PADILHA MI }\end{array}$ & $\begin{array}{l}\text { Rev. Enferm. } \\
\text { Atenção Saúde }\end{array}$ & $\begin{array}{l}\text { Analisar aspectos acerca da } \\
\text { utilização do conhecimento e } \\
\text { atitudes de estudantes de } \\
\text { enfermagem como forma de } \\
\text { prevenção a IST }\end{array}$ & $\begin{array}{c}\text { A preparação do profissional deve ser iniciada na formação } \\
\text { acadêmica, pois os conhecimentos profissionais, na } \\
\text { grande maioria das vezes, são adquiridos durante permanência } \\
\text { do indivíduo no curso de graduação }\end{array}$ \\
\hline $\begin{array}{l}\text { Adolescentes em Conflitos } \\
\text { com a Lei da Vulnerabilidade } \\
\text { para IST/HIV/AIDS }\end{array}$ & $\begin{array}{l}\text { SILVA SPC e } \\
\text { GUISANDE T }\end{array}$ & $\begin{array}{l}\text { Rev. Enferm } \\
\text { Atenção } \\
\text { Saúde(online) }\end{array}$ & $\begin{array}{l}\text { Identificar formas de exposição } \\
\text { que os adolescentes ficam frente } \\
\text { as IST's }\end{array}$ & $\begin{array}{c}\text { O início da vida sexual precoce é uma situação que } \\
\text { colaboram com o aumento do risco de vim adquirir uma IST, } \\
\text { pois estudos mostram que práticas e comportamentos de risco } \\
\text { na juventude irão refletir na vida adulta }\end{array}$ \\
\hline $\begin{array}{l}\text { Uso de preservativos por } \\
\text { alunos de cursos de saúde } \\
\text { em uma universidade pública }\end{array}$ & $\begin{array}{c}\text { TEIXEIRA RC, et } \\
\text { al. }\end{array}$ & $\begin{array}{l}\text { Semina: Ciências } \\
\text { Biológicas e da } \\
\text { saúde, londrina }\end{array}$ & $\begin{array}{c}\text { Identificar a relação com } \\
\text { preservativos dos acadêmicos de } \\
\text { saúde como forma de prevenção } \\
\text { das IST's }\end{array}$ & $\begin{array}{c}\text { A maioria dos jovens universitários possui o conhecimento } \\
\text { adequado sobre o uso de preservativos, mas uma pequena } \\
\text { minoria admite fazer relações sexuais sem o uso de } \\
\text { preservativos }\end{array}$ \\
\hline $\begin{array}{l}\text { O conhecimento e o ensino } \\
\text { sobre doenças sexualmente } \\
\text { transmissíveis entre } \\
\text { universitários }\end{array}$ & $\begin{array}{l}\text { CASTRO EL, et } \\
\text { al. }\end{array}$ & $\begin{array}{l}\text { Ciência e Saúde } \\
\text { Coletiva }\end{array}$ & $\begin{array}{l}\text { Identificar o grau de } \\
\text { conhecimento sobre as IST's } \\
\text { entre os universitários }\end{array}$ & $\begin{array}{c}\text { O conhecimento é importante na prevenção das IST's, mas não } \\
\text { basta estar ciente da necessidade de usar preservativo se } \\
\text { mesmo assim os jovens não usam }\end{array}$ \\
\hline
\end{tabular}

Fonte: Pereira R, et al., 2020. 
Atualmente, nota-se que o número de adolescentes que tem a primeira relação precocemente cresce, consequentemente há uma maior vulnerabilidade às IST's, configurando assim um sério problema de saúde pública (OLIVEIRA PS, et al., 2018). Dessa forma, acredita-se que a escola pode ser utilizada como uma estratégia para minimizar a falta de informação e a ocorrências dessas infecções na adolescência. Contudo, sabe-se que educar para sexualidade é dificil e, principalmente, com adolescentes, já que não é apenas uma transmissão de informações entre quem sabe e quem vai aprender, pois a sexualidade é algo íntimo ligada ao ser humano no interior privado, sendo esse resultado de sua cultura ou de relações pessoais estabelecidas durante sua vida (CORTEZ EA e SILVA LM, 2017).

A adolescência compreende um período de profundas transformações físicas e psicossociais, influenciadas pelo despertar da sexualidade e separação simbólica dos pais, ainda mais com as perspectivas de particularidades de vida de cada indivíduo pela fase adulta. Sentimentos de invulnerabilidade, pensamento mágico e atitudes contestadoras são algumas das características que dificultam a utilização efetiva de métodos de prevenção como os preservativos, esses fatos são contrários as recomendações sobre as práticas sexuais de maneira segura (SÁFADI MAP, 2018; VELHO PEF, 2017).

Ademais, há ainda como comportamentos de risco e fator de vulnerabilidade dos adolescentes, o uso do álcool e de outras drogas, frequentemente utilizadas, causando prejuízos de forma direta à saúde aumentando assim a vulnerabilidade as IST's (SÁFADI, 2018). Autores como Cortez EA e Silva LM (2017) ressaltam que uma das melhores maneiras de se promover educação em saúdem no que se diz respeito a sexualidade e IST's, é na escola. Entre as ações importantes para isso estão: palestras realizadas pelos profissionais de saúde, diálogo permanente entre servidores para dinamizar as IST's e rodas de conversas com os adolescentes sobre sexualidade. Assim é essencial destacar a importância das ações no âmbito educacional no que se diz respeito às IST's, como forma de passar conhecimento como prevenção.

Por meio da pesquisa, Teixeira $\mathrm{RC}$, et al. (2018) verificaram que com relação à frequência do uso de preservativos por jovens universitários, fica evidente que os mesmos possuem conhecimentos acerca da importância disso, mas que mesmo assim um pequeno grupo ainda realiza relações sexuais sem uso de preservativos. Constatando-se assim que a maioria das causas para que isso acontecesse era não possuir o preservativo no momento da relação e ter confiança no parceiro. Apesar do conhecimento ser importante, é necessário ir além de saber da importância de usar preservativo para prevenção, conhecendo, assim, as IST's para considerar as consequências e os riscos em adquiri-las (CASTRO EL, et al., 2016).

O início da vida sexual precoce é uma situação que colaboram com o aumento do risco de vim adquirir IST's, pois estudos mostram que práticas e comportamentos de risco na juventude irão refletir na vida adulta (SILVA SPC, et al., 2018). Com isso, destaca-se a importância de considerar os contextos de vulnerabilidade da população e a necessidade de ações contínuas para diminuir os índices entre essas pessoas. Nesse sentido é importante ações por parte dos universitários da área de saúde como forma de conscientização sobre formas de prevenção com os grupos mais vulneráveis (MURAD ACS, et al., 2018).

Tendo o profissional de saúde uma intrínseca ligação com o autocuidado, considerado assim como o objeto epistemológico das ciências da saúde, o que torna, consequentemente, as atividades de educação relacionadas ao autocuidado umas das competências da equipe multidisciplinar (SILVA AR e PADILHA MI, 2016).

Acerca dos conhecimentos e atitudes dos acadêmicos da área de saúde, é importante destacar que o compartilhamento de informações sobre as IST's com os grupos mais vulneráveis é uma iniciativa que colabora de forma positiva para a prevenção com aqueles que não detém determinado conhecimento (ANDRADE J, et al., 2017; MURAD ACS, et al., 2018).

Vale destacar a importância do profissional de saúde na prevenção das IST's, mas para que esse trabalho seja efetuado de maneira correta e eficiente, tais profissionais devem ter conhecimento e capacitação continuamente para essa atividade. Nesse sentido a preparação do profissional deve ser iniciada na formação acadêmica, pois os conhecimentos profissionais, na grande maioria das vezes, são adquiridos durante a permanência do indivíduo no curso de graduação (SILVA AR e PADILHA MI, 2016). 


\section{CONSIDERAÇÕES FINAIS}

As IST's representam um grande problema de saúde, sabendo que a população jovem é a mais atingida, vale ressaltar a importância de se estudar o comportamento dos jovens frente as IST's no meio acadêmico. Ao analisar isso, em especial nas áreas de saúde, evidenciou-se que eles possuem conhecimentos sobre essa problemática, como as formas de prevenção e as consequências, contudo ainda assim praticam relações sexuais de forma desprotegida ficando vulneráveis a se infectar. Torna-se imprescindível destacar que o conhecimento é importante para a prevenção, porém não é fundamental apenas estar ciente da necessidade de proteger-se, já que mesmo contendo conhecimento os acadêmicos de saúde insistem em não utilizar preservativos nas relações sexuais. Com isso, sabe-se que é preciso colocar em prática os conhecimentos adquiridos e se prevenir efetivamente com os meios existentes, bem como, por serem acadêmicos da área da saúde, possuidores de conhecimento, cabe a eles serem exemplo para os demais indivíduos da sociedade.

\section{REFERÊNCIAS}

1. ALMEIDA LCG, et al. Bonde universitário: ações de prevenção das infecções sexualmente transmissíveis: um relato de experiência. Revista Eletrônica Acervo Saúde, 2020;12(6):e3268.

2. ANDRADE J, et al. Vulnerabilidade de idosos a infecções sexualmente transmissíveis. Acta Paulista de Enfermagem, 2017;30(1):8-15.

3. BRASIL. Ministério da Saúde. Secretaria de Vigilância em Saúde. Programa Nacional de DST e Aids. Protocolo Clínico e Diretrizes Terapêuticas (Pcdt): Atenção Integral às Pessoas com Infecções Sexualmente Transmissíveis (IST). Brasília: Ministério da Saúde, 2015.

4. BRASIL. Ministério da Saúde. Secretaria de Vigilância em Saúde. Programa Nacional de DST e Aids. Protocolo Clínico e Diretrizes Terapêuticas (Pcdt): Atenção Integral às Pessoas com Infecções Sexualmente Transmissíveis (IST). Brasília: Ministério da Saúde, 2019.

5. CASTRO EL, et al. Pesquisa-Ação: Promovendo Educação em Saúde com Adolescentes Sobre Infecção Sexualmente Transmissível. Rev. Enferm UFPE online, 2017;11(9):3642-9.

6. CORTEZ EA, SILVA LM. Research-action: promoting health education with adolescents on sexually transmissible infections. Journal of Nursing UFPE, online, 2017;11(9):3642-3649.

7. MURAD ACS, et al. Experiência de Acadêmicos de Medicina na Prevenção de Infecções Sexualmente Transmissíveis. Cuid. Arte Enfermagem, 2018;12(2):200-203.

8. OLIVEIRA PS, et al. Vulnerabilidade de Adolescentes às Doenças Sexualmente Transmissíveis na Atenção Primária. Rev. Enferm UFPE online, 2018;12(3):735-762.

9. PEREIRA GFM, et al. HIV/aids, hepatites virais e outras IST no Brasil: tendências epidemiológicas. Rev. bras. epidemiol., 2019, 22(1).

10. SÁFADI MAP. Infecções Sexualmente Transmissíveis na Adolescência. SBP- Sociedade Brasileira de Pediatria, ํㅜ 6, 2018.

11. SALES WB, et al. Comportamento sexual de risco e conhecimento sobre IST/SIDA em universitários da saúde. Rev. Enf. Ref., 2016;6(10):19-27.

12. SILVA AR, PADILHA MI. Acadêmicos de Enfermagem e Seu Autocuidado em Relação a Doenças Sexualmente Transmissíveis. Rev. Enferm Atenção Saúde(Online), 2016;5(1):36-50.

13. SILVA BB, et al. "Formando multiplicadores para prevenção de infecções sexualmente transmissíveis" e impacto na formação de acadêmicos de enfermagem. Enfermagem Brasil, 2018; 15(4).

14. SILVA SPC, et al. Adolescentes em Conflitos com a Lei da Vulnerabilidade Para IST/HIV/AIDS: conhecimentos e vivências. Rev. Enferm Atenção Saúde (Online), 2018;7(2):95-108.

15. SOUZA TS, et al. Revisão Integrativa: o que é e como fazer. Einstein, 2010;8(1):102-106.

16. TEIXEIRA RC, et al. Uso de preservativos por alunos de cursos de saúde em uma universidade pública. Semina: Ciências Biológicas e da saúde, londrina, 2018; 39(1):85-90.

17. VELHO PEF. O conhecimento e o ensino sobre doenças sexualmente transmissíveis entre universitários. Ciência e Saúde Coletiva, 2016;21(6):1975-1984. 